Supporting Information for

\title{
Kinetics, Thermodynamics and Scale Up of an Azeotropic Drying Process: Mapping Rapid Phase Conversion with Process Analytical Technology
}

\author{
Zachary E. X. Dance ${ }^{1 *}$, Morgan Crawford ${ }^{2}$, Aaron Moment ${ }^{3}$, Andrew Brunskill ${ }^{4}$, Busolo \\ Wabuyele ${ }^{5}$
}

${ }^{1}$ Data Rich Measurements, Department of Analytical Research and Development, Merck Research Laboratories, Merck \& Co., Inc., Rahway, New Jersey, 07065, United States

${ }^{2}$ Department of Process Research and Development, Merck Research Laboratories, Merck \& Co., Inc., Rahway, New Jersey, 07065, United States

${ }^{3}$ Columbia University in the City of New York, Department of Chemical Engineering, $500 \mathrm{~W}$. 120th St., NY, NY 10027

${ }^{4}$ Preformulation Sciences, Merck Research Laboratories, Merck \& Co., Inc., Rahway, New Jersey, 07065, United States

${ }^{5}$ Biologics and Vaccines Analytics - Process Analytical Technology, Merck Manufacturing Division (MMD), Merck \& Co., Inc., Kenilworth, New Jersey, 07033, United States

*Email: zachary_dance@merck.com.

KEYWORDS

PAT, Crystallization, Kinetics, Spectroscopy, Thermodynamics, Phase Conversion 


\section{Supporting Figures}

Figure S1. Thermal ellipsoid representation of 2'-C-Methyluridine Anhydrate (AH) with thermal ellipsoids set at the $50 \%$ probability level. ......................................................... 3

Figure S2. Thermal ellipsoid representation of 2'-C-Methyluridine Hemihydrate (HH) with thermal ellipsoids set at the $50 \%$ probability level. A full water molecule is shown with the other half of the water molecule generated by crystallographic symmetry.

Figure S3. Thermal ellipsoid representation of 2'-C-Methyluridine Dihydrate (DH) with

thermal ellipsoids set at the $50 \%$ probability level. 7

Figure S4. TGA curves of AH, HH, and DH prior to decomposition above $200^{\circ} \mathrm{C} \ldots \ldots \ldots \ldots \ldots . . . . . .11$ Figure S5. Raman spectra corresponding to Table 2 in manuscript. Color coding matches that in Figure 6A. Raman was used to analyze the slurry samples after multiple days of equilibration at the given temperature and solids loading. The probe was inserted into the slurries and the phases present were determined using the region $1550-1750 \mathrm{~cm}^{-1}$. 12 Figure S6. IR spectra corresponding to A) MU system distillation with $5 \mathrm{~L} / \mathrm{kg} \mathbf{D H}$ (see Figure 7), and B) Acetonitrile distillation starting with $3 \mathrm{wt} \%(30 \mathrm{~K} \mathrm{ppm}) \mathrm{H}_{2} \mathrm{O}$ (see Figure 9, blue trace). The diamond absorption region $\left(1950-2350 \mathrm{~cm}^{-1}\right)$ is not shown. 13

\section{Supporting Tables}

Table S1. Crystal Data and Structure Refinement for 2' -C-Methyluridine Anhydrate (AH)...... 4 Table S2. Crystal Data and Structure Refinement for 2'-C-Methyluridine Hemihydrate (HH) .. 6 Table S3. Crystal Data and Structure Refinement for 2'-C-Methyluridine Dihydrate (DH) ....... 8 Table S4. Hydrogen bonding tables $\left[\AA \AA\right.$ and $\left.{ }^{\circ}\right]$............................................................... 9 


\section{Crystal Data and Structure Refinement for 2'-C-Methyluridine Anhydrate (AH) (CCDC 2007022)}

A single crystal grown from ethanol by solvent evaporation was selected for single crystal X-ray data analysis. The crystal was a colorless irregular cut block with dimensions of $0.3 \mathrm{~mm} \times 0.2 \mathrm{~mm}$ $\mathrm{x} 0.1 \mathrm{~mm}$. Data collection was performed on a Bruker Apex II system at $100 \mathrm{~K}$. The unit cell was determined to be orthorhombic in space group P $2{ }_{1} 2{ }_{1} 2_{1}$ and the structure contained one molecule of 2'-C-Methyluridine (MU) in the asymmetric unit and confirmed the form as anhydrous. Crystallographic data is summarized in Table S1. Absolute configuration was determined by anomalous-dispersion effects in diffraction measurements on the crystal and confirmed that the stereochemistry at all of the stereogenic centers was $R$. Figure $\mathrm{S} 1$ shows a thermal ellipsoid representation of $\mathbf{A H}$ with thermal ellipsoids set at the $50 \%$ probability level. Coordinates, refinement details and structure factors have been deposited with the Cambridge Crystallographic Data Centre (CCDC 2007022).

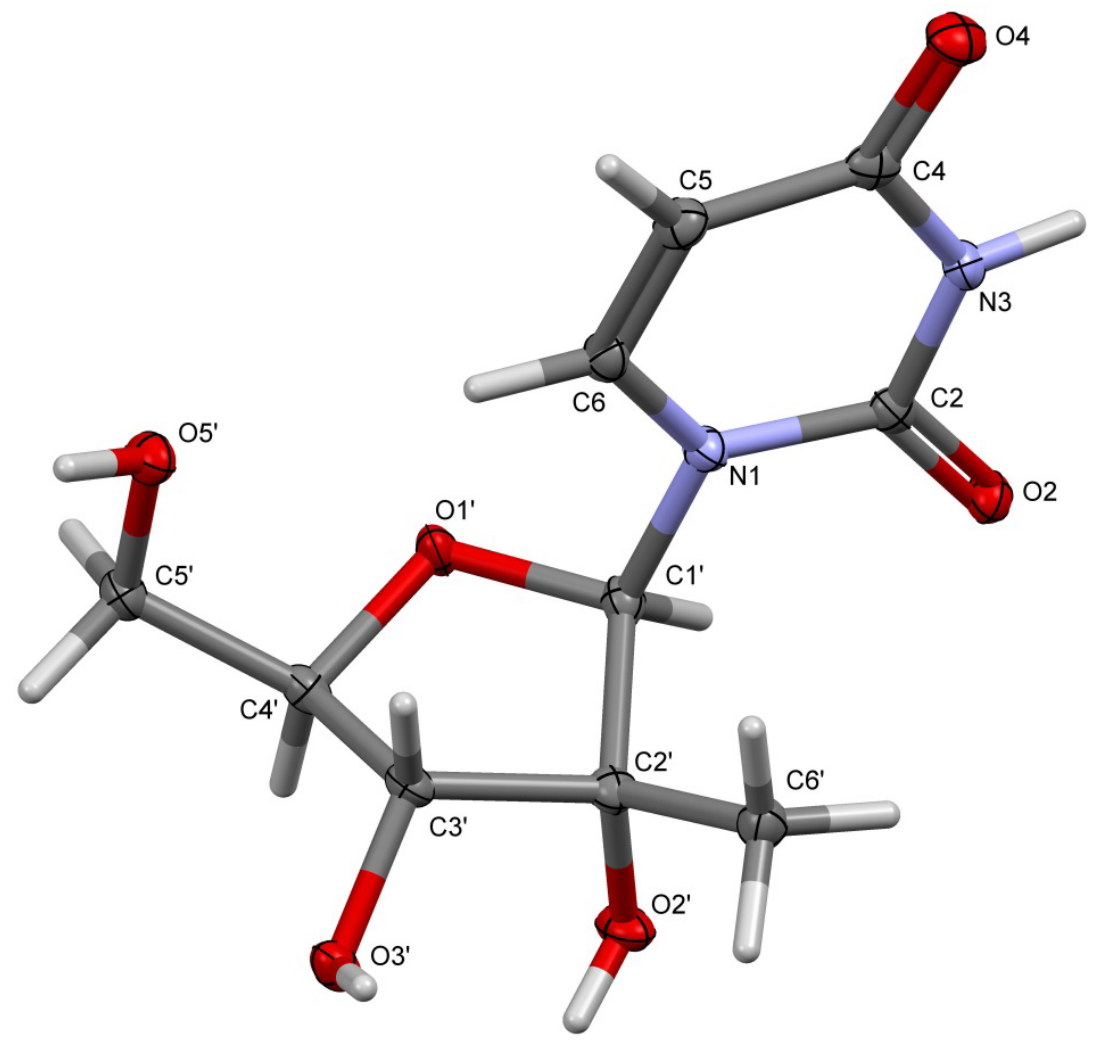

Figure S1. Thermal ellipsoid representation of 2'-C-Methyluridine Anhydrate (AH) with thermal ellipsoids set at the $50 \%$ probability level. 
Table S1. Crystal Data and Structure Refinement for 2'-C-Methyluridine Anhydrate (AH)

(CCDC 2007022)

Identification code mdj083

Empirical formula

$\mathrm{C}_{10} \mathrm{H}_{14} \mathrm{~N}_{2} \mathrm{O}_{6}$

Formula weight

258.23

Temperature

$100(2) \mathrm{K}$

Wavelength

$1.54178 \AA$

Crystal system

Orthorhombic

Space group

Unit cell dimensions

$\mathrm{P} 2{ }_{1} 2_{1} 2_{1}$

Volume

$\mathrm{a}=7.1273(4) \AA$ $\alpha=90^{\circ}$.

$\mathrm{b}=9.0099(5) \AA$ $\beta=90^{\circ}$.

$\mathrm{c}=17.1540(9) \AA$ $\gamma=90^{\circ}$.

Z $1101.57(10) \AA^{3}$

Density (calculated)

4

Absorption coefficient

$\mathrm{F}(000)$

$1.557 \mathrm{~g} / \mathrm{cm}^{3}$

$1.118 \mathrm{~mm}^{-1}$

Crystal size

544

Theta range for data collection

$0.300 \times 0.200 \times 0.100 \mathrm{~mm}^{3}$

Index ranges

5.157 to $66.558^{\circ}$.

$-8<=\mathrm{h}<=8,-10<=\mathrm{k}<=10,-20<=1<=19$

Reflections collected

7181

Independent reflections

$1946[\mathrm{R}($ int $)=0.0216]$

Completeness to theta $=66.500^{\circ}$

$99.9 \%$

Absorption correction

Semi-empirical from equivalents

Max. and min. transmission

0.894 and 0.799

Refinement method

Full-matrix least-squares on $\mathrm{F}^{2}$

Data / restraints / parameters

1946 / 0 / 177

Goodness-of-fit on $\mathrm{F}^{2}$

1.045

Final R indices [I $>2 \operatorname{sigma}(\mathrm{I})]$

$\mathrm{R} 1=0.0218, \mathrm{wR} 2=0.0572$

$\mathrm{R}$ indices (all data)

$\mathrm{R} 1=0.0219, \mathrm{wR} 2=0.0573$

Absolute structure parameter

$0.02(4)$

Extinction coefficient

$0.0041(5)$

Largest diff. peak and hole

0.206 and -0.162 e. $\AA^{-3}$ 


\section{Crystal Data and Structure Refinement for 2'-C-Methyluridine Hemihydrate (HH) (CCDC 2007023)}

A single crystal isolated from acetonitrile and water (9:1) by slurry turnover was selected for single crystal X-ray data analysis. The crystal was a colorless block with dimensions of $0.12 \mathrm{~mm} \times 0.10$ $\mathrm{mm} \times 0.08 \mathrm{~mm}$. Data collection was performed on a Bruker Apex II system at 100K. The unit cell was determined to be orthorhombic in space group $\mathrm{P} 2{ }_{1} 2_{1} 2$ and the structural asymmetric unit contained one molecule of 2'-C-Methyluridine (MU) and half a water of hydration that was located on a crystallographic two-fold axis, consistent with the assignment as a hemihydrate. Crystallographic data is summarized in Table S2. Absolute configuration was determined by anomalous-dispersion effects in diffraction measurements on the crystal and confirmed that the stereochemistry at all of the stereogenic centers was $R$. Figure S2 shows a thermal ellipsoid representation of $\mathbf{H H}$ with thermal ellipsoids set at the $50 \%$ probability level. Coordinates, refinement details and structure factors have been deposited with the Cambridge Crystallographic Data Centre (CCDC 2007023).

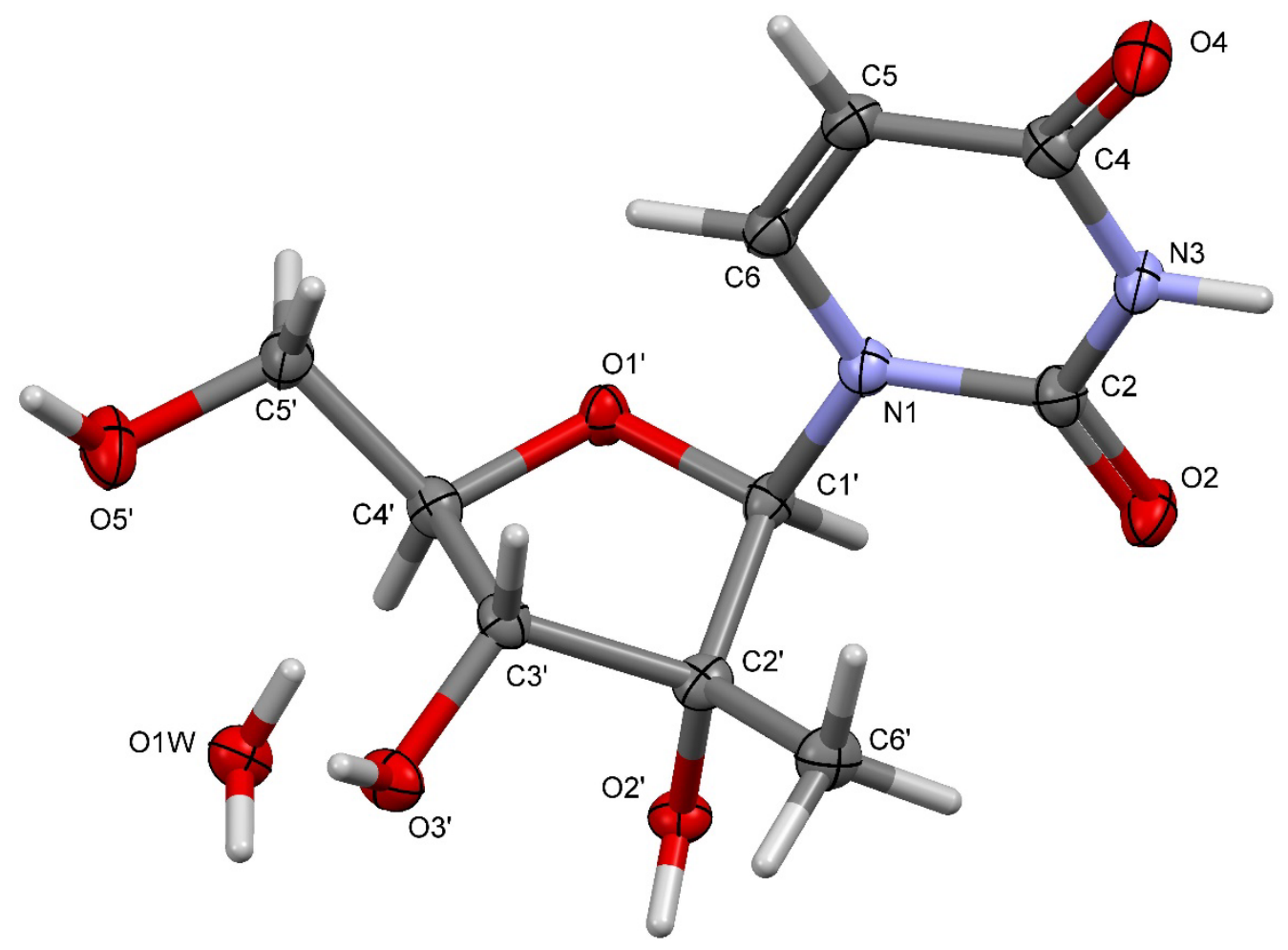

Figure S2. Thermal ellipsoid representation of 2'-C-Methyluridine Hemihydrate $(\mathbf{H H})$ with thermal ellipsoids set at the $50 \%$ probability level. A full water molecule is shown with the other half of the water molecule generated by crystallographic symmetry. 
Table S2. Crystal Data and Structure Refinement for 2'-C-Methyluridine Hemihydrate (HH)

(CCDC 2007023)

Identification code

mdk069_ab

Empirical formula

$\mathrm{C}_{10} \mathrm{H}_{14} \mathrm{~N}_{2} \mathrm{O}_{6} \cdot 0.5\left(\mathrm{H}_{2} \mathrm{O}\right)$

Formula weight

267.24

Temperature

100(2) K

Wavelength

$1.54178 \AA$

Crystal system

Orthorhombic

Space group

Unit cell dimensions

$\mathrm{P} 2{ }_{1} 22$

$\begin{array}{ll}a=7.1184(8) \AA & \alpha=90^{\circ} . \\ b=28.602(3) \AA & \beta=90^{\circ} . \\ c=5.6452(7) \AA & \gamma=90^{\circ} .\end{array}$

Volume

1149.4(2) $\AA^{3}$

$\mathrm{Z}$

4

Density (calculated)

$1.544 \mathrm{~g} / \mathrm{cm}^{3}$

Absorption coefficient

$1.124 \mathrm{~mm}^{-1}$

$\mathrm{F}(000)$

564

Crystal size

$0.12 \times 0.10 \times 0.08 \mathrm{~mm}^{3}$

Theta range for data collection

6.189 to $66.569^{\circ}$.

Index ranges

Reflections collected

$-7<=\mathrm{h}<=8,-34<=\mathrm{k}<=33,-6<=\mathrm{l}<=6$

7269

Independent reflections

$2032[\mathrm{R}(\mathrm{int})=0.0373]$

Completeness to theta $=66.500^{\circ}$

$99.8 \%$

Absorption correction

Semi-empirical from equivalents

Max. and min. transmission

0.914 and 0.808

Refinement method

Full-matrix least-squares on $\mathrm{F}^{2}$

Data / restraints / parameters

2032 / $0 / 182$

Goodness-of-fit on $\mathrm{F}^{2}$

1.109

Final $R$ indices [I $>2 \operatorname{sigma}(\mathrm{I})]$

$\mathrm{R} 1=0.0309, \mathrm{wR}_{2}=0.0809$

$\mathrm{R}$ indices (all data)

$\mathrm{R} 1=0.0314, \mathrm{wR}_{2}=0.0814$

Absolute structure parameter

$0.02(10)$

Largest diff. peak and hole

0.263 and -0.180 e. $\AA^{-3}$ 


\section{Crystal Data and Structure Refinement for 2'-C-Methyluridine Dihydrate (DH) (CCDC 2007024)}

A single crystal grown from ethanol and water (1:1) by solvent evaporation was selected for single crystal X-ray data analysis. The crystal was a colorless block with dimensions of $0.40 \mathrm{~mm} \times 0.40$ $\mathrm{mm} \times 0.35 \mathrm{~mm}$. Data collection was performed on a Bruker Apex II system at $100 \mathrm{~K}$. The unit cell was determined to be orthorhombic in space group P2 ${ }_{1} 2{ }_{1} 2_{1}$ and the structure contained one molecule of 2'-C-Methyluridine (MU) and two waters of hydration in the asymmetric unit and confirmed the form as dihydrate. Crystallographic data is summarized in Table S3. Absolute configuration was determined by anomalous-dispersion effects in diffraction measurements on the crystal and confirmed that the stereochemistry at all of the stereogenic centers was $R$. Figure S3 shows a thermal ellipsoid representation of $\mathbf{D H}$ with thermal ellipsoids set at the $50 \%$ probability level. Coordinates, refinement details and structure factors have been deposited with the Cambridge Crystallographic Data Centre (CCDC 2007024).

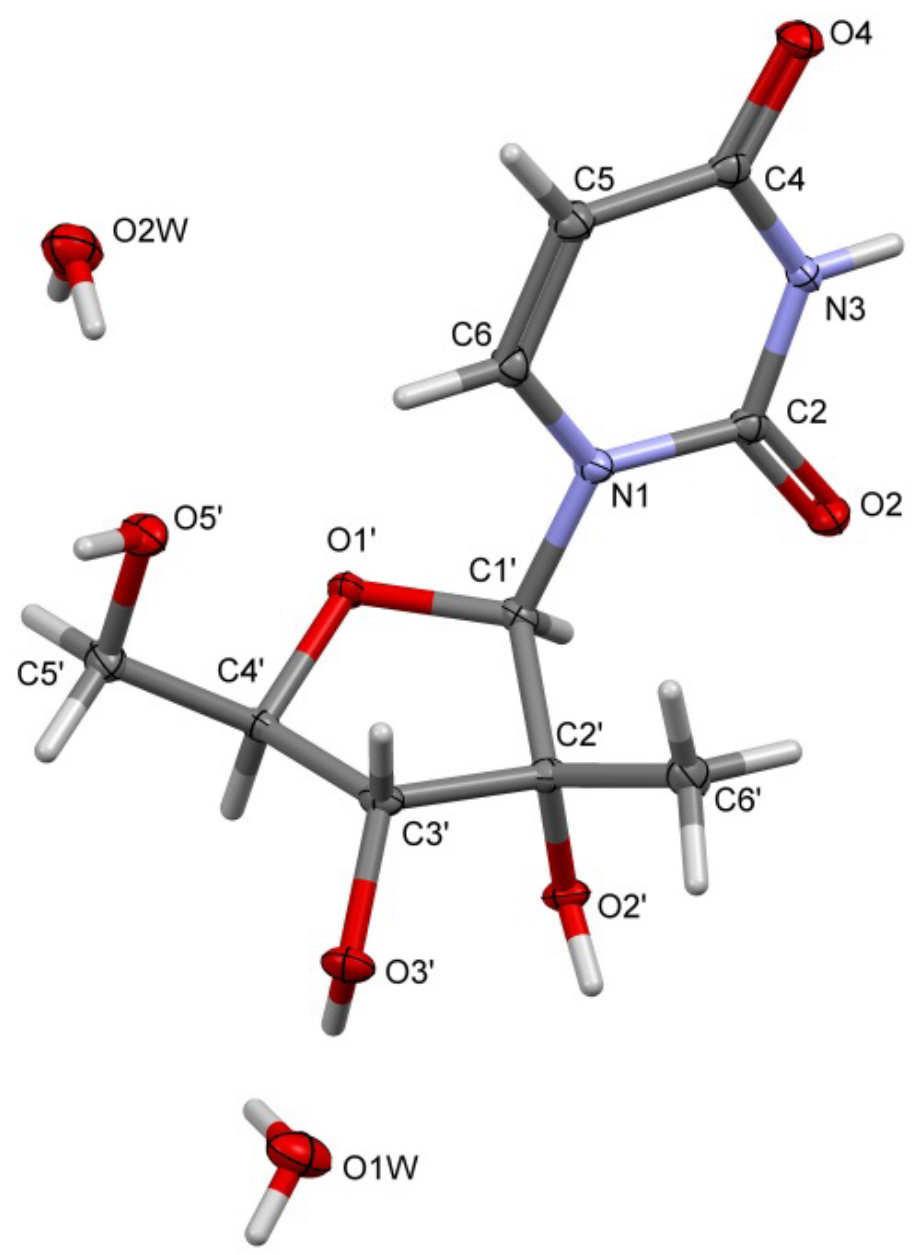

Figure S3. Thermal ellipsoid representation of 2'-C-Methyluridine Dihydrate (DH) with thermal ellipsoids set at the $50 \%$ probability level. 
Table S3. Crystal Data and Structure Refinement for 2'-C-Methyluridine Dihydrate (DH)

(CCDC 2007024)

Identification code

mdj082

Empirical formula

$\mathrm{C}_{10} \mathrm{H}_{14} \mathrm{~N}_{2} \mathrm{O}_{6} \cdot 2\left(\mathrm{H}_{2} \mathrm{O}\right)$

Formula weight

294.26

Temperature

$100(2) \mathrm{K}$

Wavelength

$1.54178 \AA$

Crystal system

Orthorhombic

Space group

Unit cell dimensions

$\mathrm{P} 2{ }_{1} 2_{1} 2_{1}$

$\begin{array}{ll}\mathrm{a}=7.2564(6) \AA & \alpha=90^{\circ} . \\ \mathrm{b}=10.1171(8) \AA & \beta=90^{\circ} . \\ \mathrm{c}=17.2505(14) \AA & \gamma=90^{\circ} .\end{array}$

Volume

1266.42(18) $\AA^{3}$

Z

4

Density (calculated)

$1.543 \mathrm{~g} / \mathrm{cm}^{3}$

Absorption coefficient

$1.165 \mathrm{~mm}^{-1}$

$\mathrm{F}(000)$

624

Crystal size

$0.400 \times 0.400 \times 0.350 \mathrm{~mm}^{3}$

Theta range for data collection

5.068 to $66.548^{\circ}$.

Index ranges

$-8<=\mathrm{h}<=8,-11<=\mathrm{k}<=7,-20<=1<=20$

Reflections collected

8169

Independent reflections

$2213[\mathrm{R}(\mathrm{int})=0.0229]$

Completeness to theta $=66.500^{\circ}$

$99.3 \%$

Absorption correction

Semi-empirical from equivalents

Max. and min. transmission

0.665 and 0.610

Refinement method

Full-matrix least-squares on $\mathrm{F}^{2}$

Data / restraints / parameters

$2213 / 0 / 207$

Goodness-of-fit on $\mathrm{F}^{2}$

1.079

Final $\mathrm{R}$ indices [I $>2 \operatorname{sigma}(\mathrm{I})]$

$\mathrm{R} 1=0.0226, \mathrm{wR} 2=0.0602$

$\mathrm{R}$ indices (all data)

$\mathrm{R} 1=0.0226, \mathrm{wR} 2=0.0602$

Absolute structure parameter

$-0.02(3)$

Extinction coefficient

$0.0121(9)$

Largest diff. peak and hole

0.251 and -0.176 e. $\AA^{-3}$ 
Table S4. Hydrogen bonding tables $\left[\AA\right.$ and $\left.{ }^{\circ}\right]$

\section{Anhydrate}

\begin{tabular}{lcccc}
\hline $\mathrm{D}-\mathrm{H} . . . \mathrm{A}$ & $\mathrm{d}(\mathrm{D}-\mathrm{H})$ & $\mathrm{d}(\mathrm{H} \ldots \mathrm{A})$ & $\mathrm{d}(\mathrm{D} \ldots \mathrm{A})$ & $<(\mathrm{DHA})$ \\
\hline $\mathrm{N}(3)-\mathrm{H}(3) \ldots \mathrm{O}\left(3^{\prime}\right) \# 1$ & $0.86(2)$ & $2.07(2)$ & $2.917(2)$ & $170(2)$ \\
$\mathrm{O}\left(2^{\prime}\right)-\mathrm{H}\left(2^{\prime}\right) \ldots \mathrm{O}\left(1^{\prime}\right) \# 2$ & $0.78(3)$ & $2.65(3)$ & $3.3656(17)$ & $152(2)$ \\
$\mathrm{O}\left(3^{\prime}\right)-\mathrm{H}\left(3^{\prime}\right) \ldots \mathrm{O}\left(5^{\prime}\right) \# 3$ & $0.82(2)$ & $2.04(3)$ & $2.8291(18)$ & $161(2)$ \\
$\mathrm{O}\left(5^{\prime}\right)-\mathrm{H}\left(5^{\prime}\right) \ldots \mathrm{O}(4) \# 4$ & $0.79(3)$ & $1.98(3)$ & $2.7507(17)$ & $164(2)$ \\
& & & & \\
\hline
\end{tabular}

Symmetry transformations used to generate equivalent atoms:

$\# 1-x+1 / 2,-y+1, z+1 / 2 \quad \# 2-x+1, y-1 / 2,-z+1 / 2 \quad \# 3-x, y-1 / 2,-z+1 / 2$

$\# 4-x-1 / 2,-y+1, z-1 / 2$

\section{Hemihydrate}

\begin{tabular}{lcccc}
\hline $\mathrm{D}-\mathrm{H} \ldots \mathrm{A}$ & $\mathrm{d}(\mathrm{D}-\mathrm{H})$ & $\mathrm{d}(\mathrm{H} \ldots \mathrm{A})$ & $\mathrm{d}(\mathrm{D} \ldots \mathrm{A})$ & $<(\mathrm{DHA})$ \\
\hline $\mathrm{N}(3)-\mathrm{H}(3) \ldots \mathrm{O}(4) \# 2$ & $0.86(4)$ & $1.94(4)$ & $2.797(3)$ & $178(3)$ \\
$\mathrm{O}\left(2^{\prime}\right)-\mathrm{H}\left(2^{\prime}\right) \ldots \mathrm{O}\left(3^{\prime}\right) \# 3$ & $0.82(4)$ & $1.91(4)$ & $2.700(3)$ & $161(3)$ \\
$\mathrm{O}\left(3^{\prime}\right)-\mathrm{H}\left(3^{\prime}\right) \ldots \mathrm{O}(1 \mathrm{~W})$ & $0.83(4)$ & $1.90(4)$ & $2.730(3)$ & $176(4)$ \\
$\mathrm{O}\left(5^{\prime}\right)-\mathrm{H}\left(5^{\prime}\right) \ldots \mathrm{O}\left(2^{\prime}\right) \# 4$ & 0.84 & 1.86 & $2.690(3)$ & 169.4 \\
$\mathrm{O}(1 \mathrm{~W})-\mathrm{H}(1 \mathrm{~W} 1) \ldots \mathrm{O}\left(5^{\prime}\right) \# 5$ & $0.80(3)$ & $1.92(3)$ & $2.712(3)$ & $173(4)$ \\
& & & & \\
\hline
\end{tabular}

Symmetry transformations used to generate equivalent atoms:

$\# 1-x,-y+2, z \quad \# 2 x+1 / 2,-y+3 / 2,-z \quad \# 3-x+1,-y+2, z$

\#4 x-1,y,z \#5 -x,-y+2,z-1 


\section{Dihydrate}

\begin{tabular}{lcccc}
\hline $\mathrm{D}-\mathrm{H} \ldots \mathrm{A}$ & $\mathrm{d}(\mathrm{D}-\mathrm{H})$ & $\mathrm{d}(\mathrm{H} \ldots \mathrm{A})$ & $\mathrm{d}(\mathrm{D} \ldots \mathrm{A})$ & $<(\mathrm{DHA})$ \\
\hline $\mathrm{N}(3)-\mathrm{H}(3) \ldots \mathrm{O}(4) \# 1$ & $0.84(3)$ & $1.98(3)$ & $2.814(2)$ & $172(2)$ \\
$\mathrm{O}\left(2^{\prime}\right)-\mathrm{H}\left(2^{\prime}\right) \ldots \mathrm{O}\left(3^{\prime}\right) \# 2$ & $0.82(3)$ & $1.87(3)$ & $2.6581(19)$ & $159(2)$ \\
$\mathrm{O}\left(3^{\prime}\right)-\mathrm{H}\left(3^{\prime}\right) \ldots \mathrm{O}(2 \mathrm{~W})$ & $0.88(3)$ & $1.80(3)$ & $2.677(2)$ & $176(2)$ \\
$\mathrm{O}\left(5^{\prime}\right)-\mathrm{H}\left(5^{\prime}\right) \ldots \mathrm{O}\left(2^{\prime}\right) \# 3$ & $0.83(3)$ & $1.89(3)$ & $2.7104(19)$ & $173(3)$ \\
$\mathrm{O}(2 \mathrm{~W})-\mathrm{H}(2 \mathrm{~W} 1) \ldots \mathrm{O}(1 \mathrm{~W}) \# 4$ & $0.75(3)$ & $2.00(3)$ & $2.739(2)$ & $166(3)$ \\
$\mathrm{O}(2 \mathrm{~W})-\mathrm{H}(2 \mathrm{~W} 2) \ldots \mathrm{O}(4) \# 5$ & $0.86(3)$ & $2.20(3)$ & $3.043(2)$ & $167(3)$ \\
$\mathrm{O}(1 \mathrm{~W})-\mathrm{H}(1 \mathrm{~W} 1) \ldots \mathrm{O}(2) \# 5$ & $0.82(4)$ & $2.40(4)$ & $3.210(2)$ & $172(3)$ \\
$\mathrm{O}(1 \mathrm{~W})-\mathrm{H}(1 \mathrm{~W} 2) \ldots \mathrm{O}\left(5^{\prime}\right)$ & $0.81(3)$ & $1.98(3)$ & $2.782(2)$ & $172(3)$ \\
& & & & \\
\hline
\end{tabular}

Symmetry transformations used to generate equivalent atoms:

$\# 1 \mathrm{x}+1 / 2,-\mathrm{y}+1 / 2,-\mathrm{z}+1 \quad \# 2 \mathrm{x}+1 / 2,-\mathrm{y}+1 / 2,-\mathrm{z} \quad \# 3 \mathrm{x}-1, \mathrm{y}, \mathrm{z}$

\#4 -x-1/2,-y+1,z-1/2 \#5-x,y+1/2,-z+1/2 


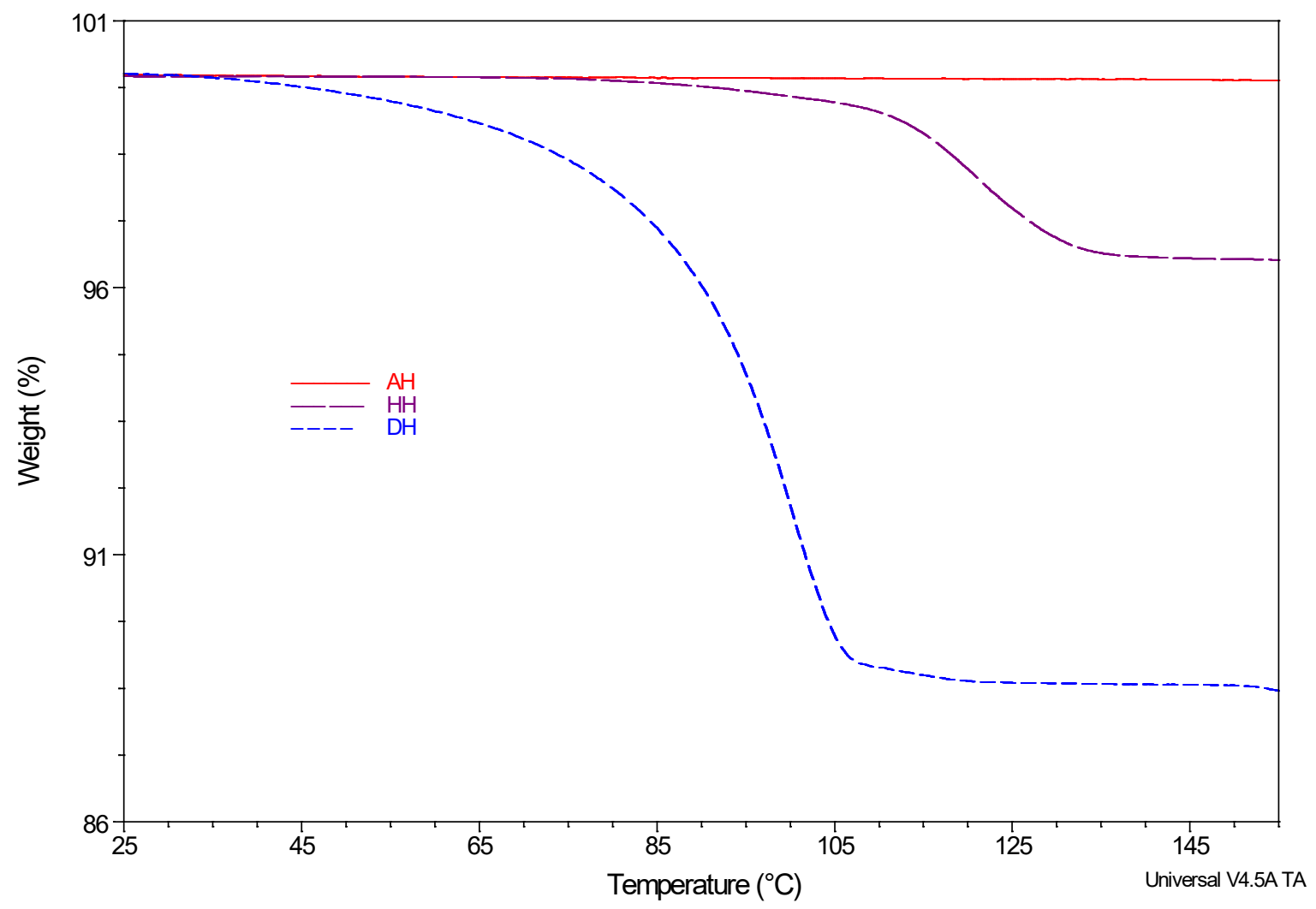

Figure S4. TGA curves of $\mathbf{A H}, \mathbf{H H}$, and $\mathbf{D H}$ prior to decomposition above $200^{\circ} \mathrm{C}$. 


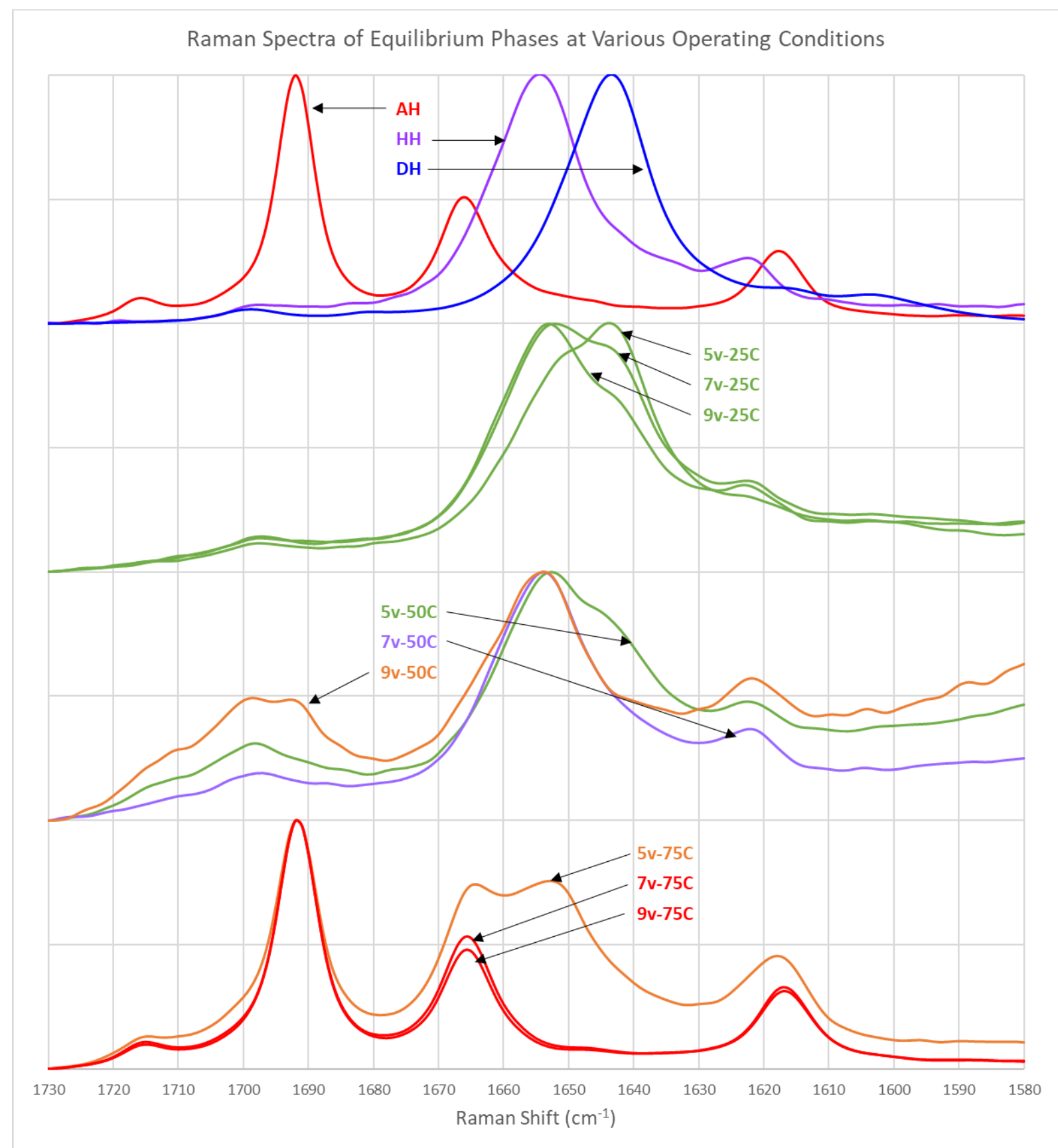

Figure S5. Raman spectra corresponding to Table 2 in manuscript. Color coding matches that in Figure 6A. Raman was used to analyze the slurry samples after multiple days of equilibration at the given temperature and solids loading. The probe was inserted into the slurries and the phases present were determined using the region $1550-1750 \mathrm{~cm}^{-1}$. 


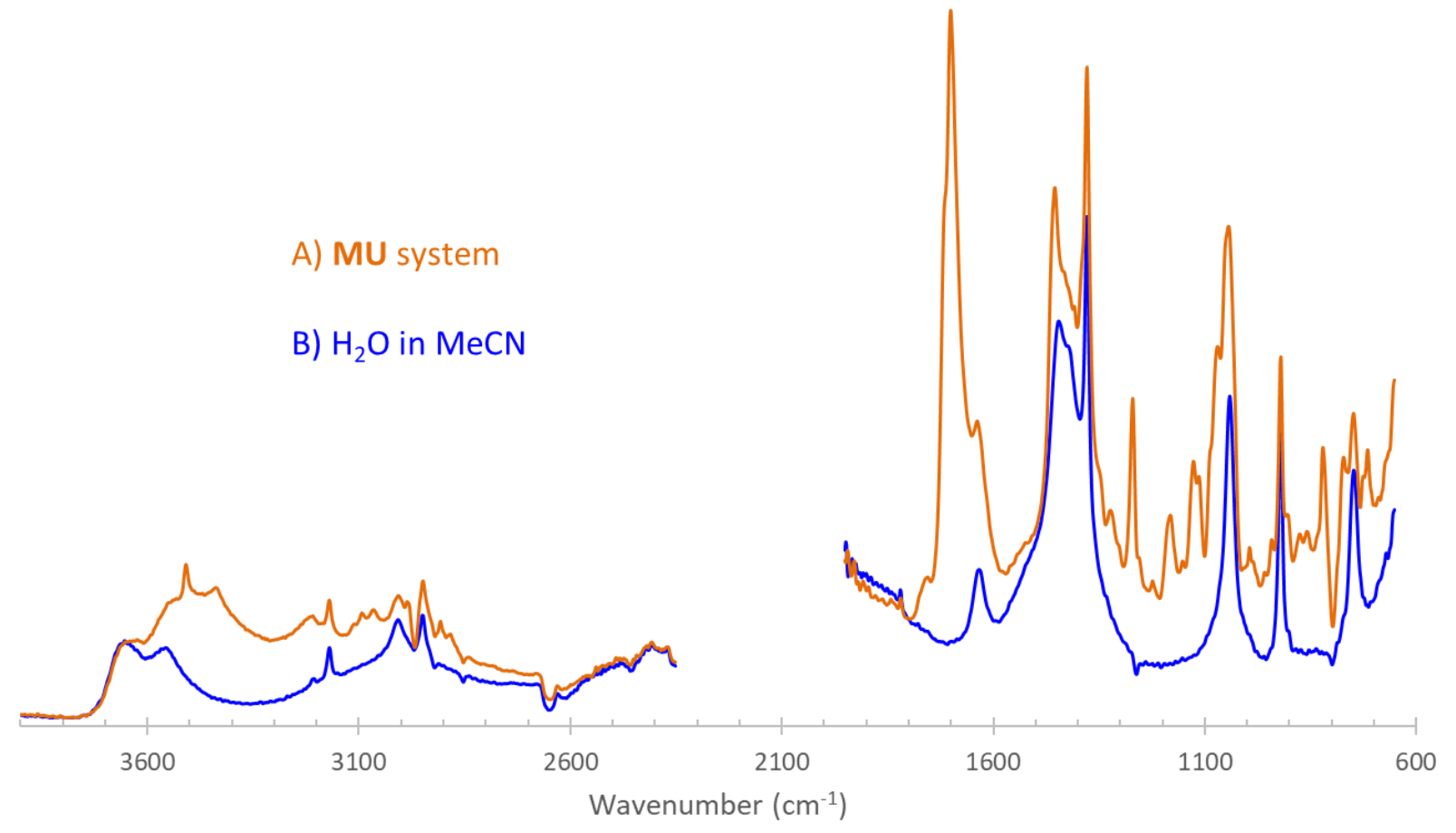

Figure S6. IR spectra corresponding to A) MU system distillation with $5 \mathrm{~L} / \mathrm{kg}$ DH (see Figure 7), and B) Acetonitrile distillation starting with $3 \mathrm{wt} \%$ (30K ppm) $\mathrm{H}_{2} \mathrm{O}$ (see Figure 9, blue trace). The diamond absorption region $\left(1950-2350 \mathrm{~cm}^{-1}\right)$ is not shown. 